\title{
Facilitators of and barriers to accessing clinical prevention services for the South Asian population in Surrey, British Columbia: a qualitative study
}

\author{
Sanaa Majid BHSc, Rachel Douglas MPH, Victoria Lee MD MPH, Elizabeth Stacy MA, \\ Arun K. Garg MD PhD, Kendall Ho MD
}

\section{Abstract}

Background: British Columbia falls short in uptake of recommended clinical prevention services, with even lower rates among immigrant populations. This study explored facilitators of and barriers to uptake of clinical prevention services among people from South Asia, who represent $31 \%$ of the population in Surrey, British Columbia.

Methods: We used a qualitative descriptive approach and employed vignettes in a focus group setting to elicit perspectives of South Asian people on accessing clinical prevention services. Participants aged 40 years or more were recruited between October 2014 and February 2015 from health care and community settings such as older-adult housing, day programs and health education events. Letters of introduction to the study were provided in English or Punjabi or both to all potential participants. We conducted qualitative content analysis of the results.

Results: Sixty-two South Asian adults (36 women and 26 men) aged 40-87 years participated in 1 of 8 focus groups in health care or community settings. Facilitators of and barriers to accessing clinical prevention services were noted at the patient, primary care provider and health care system levels. Facilitators at the patient level included taking ownership over one's health, health literacy and respecting the provider's advice; barriers included fear of the diagnosis, death and/or procedures, perceived low risk of disease or utility of the intervention, and side effects of procedures. Provider factors centred on a trust-based patient-provider relationship, strong communication and adequate time during visits. Health care system factors included such facilitators as processes to routinely offer prevention services as part of other health care or social services, systems that encourage prevention-oriented family practice and services at low or no cost to the patient.

Interpretation: Our findings validate previously identified facilitators of and barriers to accessing preventive care for immigrant populations. However, the results suggest that system-level factors influencing the duration of primary care visits may have a more salient impact on uptake of clinical prevention services in this population.

$\mathrm{P}$ opulation health improvement is a priority for British Columbia's health care system, and integrated and systematic provision of clinical prevention services is identified as a key strategy to achieve this goal. ${ }^{1-3}$ In 2009, the province's Clinical Prevention Policy Review Committee released a Lifetime Prevention Schedule, which identified priority clinical prevention activities based on clinical effectiveness, impact on population health, clinically preventable burden and cost-effectiveness. ${ }^{3}$ The following 10 activities were identified as priorities for adults: smoking cessation; alcohol screening and brief counselling; hypertension and cholesterol screening and treatment; colorectal, breast and cervical cancer screening; and influenza, pneumococcal and tetanus vaccinations. For several of these services, uptake rates in British Columbia lag behind international gold standards, ${ }^{3}$ and evidence from Canada and the United States suggests even lower uptake among ethnic minority groups, including people from South Asia. ${ }^{4-6}$ The
South Asian population is also at higher risk than the white population for both diabetes and cardiovascular disease., ${ }^{7,8}$

Despite increasing research on clinical prevention for people from South Asia, ${ }^{9-16}$ there are significant gaps in understanding barriers to service uptake in this population. Prevention programs tailored specifically to the needs of South Asian people in Canada have been unsuccessful in engaging the population in a sustainable way. ${ }^{5,10,13}$ Moreover, most studies have focused on 1 or 2 clinical prevention activities rather than

\section{Competing interests: None declared.}

This article has been peer reviewed.

Correspondence to: Rachel Douglas, rachel.douglas@fraserhealth.ca CMAJ Open 2016. DOI:10.9778/cmajo.20150142 
the full range of priority manoeuvres for adults. ${ }^{6,10,11,16} \mathrm{We}$ sought to develop a more in-depth understanding of the facilitators of and barriers to uptake of clinical prevention services for the South Asian population.

\section{Methods}

\section{Design}

We engaged a Fraser Health librarian to validate and ensure the comprehensiveness of our literature review before we developed the protocol for the study (available on request). We selected a qualitative design ${ }^{17}$ for the study to gain an indepth understanding that could inform future work. We involved health care system (e.g., Fraser Health's Diversity Services) and community (e.g., various nonprofit organizations that serve South Asian immigrant populations) partners at several stages of project design and implementation. The partners contributed to the design of data collection tools by reviewing the study protocol and all tools, assisting with recruitment and communication strategies, and facilitating logistics to integrate study sessions into their programming. This led to a more feasible and culturally appropriate design.

Data were collected through semistructured focus groups. We organized participants into groups based on sex, as recommended by our community partners. We originally designed the study to host separate focus groups for older ( $\geq 65 \mathrm{yr}$ ) and younger (40-64 yr) participants in order to focus on topics that were recommended for the group based on age and sex. However, many focus groups were integrated into ongoing community programs where it would have been inappropriate to deny participation by those outside of the designated age range, so we decided to group the participants by sex only.

We obtained background data from most participants on their enrolment into the study, using a brief demographic questionnaire adapted from the Canadian Community Health Survey. ${ }^{18}$ Background questions elicited information on age, sex, language(s) spoken, length of time in Canada, native country/region, religious beliefs, education level and level of identification with South Asian culture (using an adaptation of the South Asian Identity Scale ${ }^{9}$ ) (Appendix 1, available at www.cmajopen.ca/content/4/3/E390/suppl/DC1).

\section{Participant recruitment}

Our recruitment target was 110 South Asian (defined as people whose ethnic roots originate from the Indian subcontinent [India, Pakistan, Sri Lanka, Nepal, Bangladesh, Maldives and Bhutan]) participants based on a matrix developed to ensure that each age and sex group discussed all topics that were relevant for them. We limited study participation to people aged 40 years or more because half of the interventions discussed apply only to this age group. We used purposive sampling to include the perspectives of both younger and older women and men. We used multiple recruitment sites and mechanisms to reach a broad spectrum of the population between October 2014 and February 2015. We drew the study sample from patients and visitors to the Jim Pattison Outpatient Care and
Surgery Centre, in Surrey, British Columbia, and community settings. At the Jim Pattison Outpatient Care and Surgery Centre, we used multilingual volunteers to recruit face to face in waiting areas and a database of recently seen patients who had consented to be contacted for research opportunities. In the community, we recruited people who approached our booth at health care events and introduced the study to groups at programs and housing for older adults. We also worked with our community partners to spread the word, had volunteers in primary care waiting areas and placed posters in recreational settings. Volunteers conducting recruitment attended a training session and received a manual detailing the process and wording to be used. Letters of introduction to the study were provided in English or Punjabi or both to all potential participants. Recruitment at group events was supervised by R.D.

\section{Data collection}

We conducted focus groups in person in community settings and at the Jim Pattison Outpatient Care and Surgery Centre between December 2014 and March 2015 using the focus group moderator's guide (Appendix 1). Groups were led by a sex-matched community-based bilingual (Punjabi and English) facilitator. Both facilitators had substantial experience in conducting focus groups in community settings. Volunteers cofacilitated the groups and took field notes. R.D., who has training in group facilitation, has conducted patient interviews and has cofacilitated group therapy for health research, was an observer for all focus groups. Focus group facilitators participated in an orientation session, and a focus group was roleplayed with R.D. so that the facilitators had a chance to practise and identify any areas where they had questions.

We held the focus groups in Punjabi and provided simultaneous whisper translation of the questions and discussion into English for those who did not speak Punjabi. The team also had the capacity to offer interpretation into other South Asian languages, but this service was not needed. We recorded both the English and the Punjabi dialogue. The English-language recordings were transcribed for data analysis, and a bilingual member of the research team (S.M.) compared the transcripts to the Punjabi recordings and added notes to the transcripts regarding any dialogue that was missed or misinterpreted. Most of these notes were errors of omission and did not substantially change the meaning of the dialogue but, rather, enriched it.

We used fictitious vignettes ${ }^{19}$ to encourage in-depth discussion without the participants' having to share personally sensitive information. In each vignette, the character learns about a clinical prevention activity that he or she is eligible and insured for under British Columbia's universal health plan but does not follow through with the activity. Focus group participants were asked what the character might be thinking or feeling about the prevention activity, what might prevent him or her from accessing the service, where they might seek information about the topic and how services could change to improve accessibility.

Each focus group was assigned 3 of the 10 priority clinical prevention topics. Groups ran for a total of 90 minutes, 
with about 20 minutes allocated for each topic; the remaining time was devoted to introductions, a quick ice-breaker and breaks.

\section{Analysis}

Initial analysis focused on identifying facilitators of and barriers to accessing clinical prevention services from the patient's perspective. We conducted a qualitative content analysis and used the study objectives and research questions to develop the initial codebook. The initial coding structure was also informed by the literature on barriers to and facilitators of screening among newcomer populations ${ }^{16}$ and in the overall population of British Columbia. ${ }^{3}$

Two coders (S.M. and R.D.) worked independently using NVivo version 10 software to attach labels to parts of the text relating to the themes identified. They created additional codes and subcodes throughout this process and kept a shared $\log$ of new codes and their descriptions. The coders met after coding the first focus group transcript to review their understanding of each code and explore any discrepancies in how they had been applied. The results of the initial analysis were shared with the research team, and the ensuing discussion helped to guide subsequent coding. Coding continued with a second level of analysis to map relations between the codes and identify any emerging themes until data saturation was achieved.

It was not feasible to return the transcripts of the focus groups to participants because many of them were not literate in either English or Punjabi. However, the preliminary findings were discussed with a group of study participants and other community members at a debriefing session.

The Fraser Health Research Ethics Board approved the study, including all revisions to the protocol.

\section{Results}

A total of 81 participants were recruited for the study, of whom 76 completed a demographic interview and 62 attended 1 of the 8 focus groups ( 5 for women and 3 for men). The 62 participants represented $56 \%$ of our recruitment target of 110 participants. However, given that the age groups were collapsed, each relevant topic was discussed in all focus groups at least once. There were 6 to 10 participants per focus group. No repeat interviews or focus groups were conducted. There were no study dropouts, but some people could not be scheduled to participate in a focus group owing to logistical considerations. Four of the 5 participants without demographic interviews were from 1 of the male groups, for which the site leader requested that the focus group be done in advance of the interviews for logistical reasons; the fifth participant was a friend of a registered participant who provided consent at the focus group session. Attempts to follow up with these 5 participants by telephone were unsuccessful. The participants' demographic characteristics are summarized in Table 1. In comparison to population-based estimates for the South Asian population in Surrey,,$^{20}$ our sample had a higher proportion of people who spoke unofficial languages at home, including Punjabi $(90 \%$ v.
$81 \%)$, Hindi ( $41 \%$ v. $22 \%$ ) and Urdu (16\% v. $5 \%)$. This was also true for comparisons with those born outside of Canada (100\% v. $56 \%)$; we calculated this value using the ratio of Surrey immigrants reporting South Asian countries of origin (only India, Pakistan and Sri Lanka were available) to the number of people reporting South Asian ancestry.

\section{Facilitators of and barriers to accessing clinical prevention services}

The main findings of the study are summarized in Box 1. Facilitators of and barriers to accessing clinical prevention services appeared in 3 contexts: at the patient level, based on personal characteristics or experiences; at the provider level, based on factors related to the patient-provider interaction; and at the health care system level, based on how services are structured or delivered. Some facilitators and barriers involved more than 1 context and were coded as such. The key themes identified are given in Table 2. Table 3 provides illustrative quotes for selected themes. The final coding tree is given in Appendix 2 (available at www.cmajopen.ca/content/4/3/E390/suppl/DC1).

\section{Patient factors}

Participants stressed the importance of taking responsibility for one's own health by asking for services, advocating for health needs and keeping notes or records. Participants also identified knowledge as a key facilitator, including information about disease risks and the risks and benefits of prevention services. They recommended outreach education and collaboration with the South Asian media to increase awareness. Finally, participants mentioned culturally based respect for the advice of the health care provider as a facilitator of accessing services.

Participants identified fear as the primary barrier to uptake of services at the patient level. This included fear of diagnosis, death and procedures along with fear of the provider's recommendations and subsequent impacts on life (e.g., needing to take time off work or change dietary habits). Moreover, some participants believed that the stress of learning about a diagnosis could actually exacerbate symptoms and make one's health worse. A second barrier was perceived low risk of disease or utility of the intervention (e.g., not experiencing symptoms, living a healthy lifestyle, not seeing the need for the service). Side effects of clinical prevention activities, real or perceived, emerged as a third barrier.

\section{Provider factors}

At the primary care provider level, participants mentioned the following facilitators: active listening, whereby the participant feels the doctor is listening to him or her and acknowledging concerns; a trusting relationship, with strong provider-patient rapport; and an unhurried pace, with time for the patient to voice concerns and ask questions, and for the provider to explain procedures or changes.

Barriers at this level included not sharing information (e.g., not providing advice on relevant prevention activities, providing medication without counselling), broken trust as a result of inaction or a missed diagnosis, and rushing/not listening. 


\section{Health care system factors}

The most frequently mentioned facilitators at the health care system level included processes whereby reminders or requirements for prevention services are integrated into other services and systems (e.g., reminders for mammography, medical examination requirements for a job); availability of services, including mobile mammography, the nurse telephone hotline and influenza vaccination at pharmacies; and clinical prevention services at low or no cost to the patient.

Barriers at this level were policies that limit physicians' time with patients or limit the number of issues discussed per visit or both, conflicting or changing guidelines about a procedure and a lack of reminders for prevention services.

\section{Most important barrier}

The most strongly voiced barrier was the lack of effective communication and trust with primary care providers owing to curtailed consultations. The relative importance of this finding was confirmed at the community debriefing session, where participants endorsed community outreach education but reiterated their pressing concern about limited opportunities for discussion with primary care providers and short visit duration.

\begin{tabular}{|c|c|c|c|c|}
\hline Characteristic & $\begin{array}{l}\text { No. }(\%) \text { of } \\
\text { participants }\end{array}$ & Range & Median & Mean \\
\hline \multicolumn{5}{|l|}{ Sex, $n=62$} \\
\hline Female & $36(58)$ & & & \\
\hline Male & $26(42)$ & & & \\
\hline Age, yr, $n=58$ & & $40-87$ & 68 & 66 \\
\hline \multicolumn{5}{|l|}{ Language(s) spoken, ${ }^{*} n=58$} \\
\hline Punjabi & $52(90)$ & & & \\
\hline English & $32(55)$ & & & \\
\hline Hindi & $24(41)$ & & & \\
\hline Urdu & $9(16)$ & & & \\
\hline Other & $3(5)$ & & & \\
\hline \multicolumn{5}{|l|}{ Country of birth, $n=58$} \\
\hline India & $49(84)$ & & & \\
\hline Pakistan & $6(10)$ & & & \\
\hline Other & $3(5)$ & & & \\
\hline Years in Canada, $n=58$ & & $1-6$ & $18 \dagger$ & 19 \\
\hline \multicolumn{5}{|l|}{ Religious affiliation, $n=57$} \\
\hline Sikhism & $43(75)$ & & & \\
\hline Other & $8(14)$ & & & \\
\hline None & $6(10)$ & & & \\
\hline \multicolumn{5}{|l|}{ Highest level of education, $n=57$} \\
\hline No formal education & $8(14)$ & & & \\
\hline Elementary school & $22(39)$ & & & \\
\hline High school/other diploma & $9(16)$ & & & \\
\hline University degree or higher & $18(32)$ & & & \\
\hline \multicolumn{5}{|c|}{ Annual household income, $\$, n=26$} \\
\hline$<40000$ & $21(81)$ & & & \\
\hline$\geq 40000$ & 5 (19) & & & \\
\hline $\begin{array}{l}\text { No. of items endorsed on South } \\
\text { Asian Identity Scale, } \neq n=58\end{array}$ & & $7-10$ & 9 & 9 \\
\hline \multicolumn{5}{|c|}{$\begin{array}{l}\text { *Some participants spoke more than } 1 \text { language; all spoke either English or Punjabi. } \\
\text { †The median varied substantially from focus group to focus group (from } 5 \text { years in groups } 1 \text { and } 9 \text { [female] to } 21 \\
\text { years in group } 4 \text { [male]). } \\
\text { fOut of } 10 .\end{array}$} \\
\hline
\end{tabular}




\section{OPEN}

\section{Interpretation}

The literature on access to primary care services, including prevention services, by South Asian people in North America highlights the impact of culturally related ${ }^{9}$ and pragmatic ${ }^{10}$ barriers to uptake such as transportation and hours of service. It also describes the central role of culture and health literacy in preventive service uptake and chronic disease management for people from South Asia ${ }^{21-23}$ and other immigrant and older populations. ${ }^{24-26}$ All of these themes also emerged in our study.

However, although culturally specific and patient level barriers were present in our study, their relative importance was less than that described in the literature. The most salient barriers to accessing clinical prevention services were related to short duration of consultations and poor-quality patient-provider interactions. Moreover, this persisted when the participant's language and cultural background matched that of his or her care pro-

\section{Box 1: Summary of main findings}

Primary care transformation is an equity issue: The current model of primary care that favours short visits and 1 issue per visit disadvantages some populations more than others

Providing culturally appropriate services is necessary but not sufficient to support better health outcomes for the South Asian population: Study participants recommended culturally specific changes to service design and health education but stressed the characteristics of interactions with providers as the primary barriers to uptake of clinical prevention services

Addressing clinical prevention requires interventions in multiple contexts: Participants recommended increased community outreach and education, policies to improve provider interactions and health care system changes to fully address their concerns

Table 2: Summary of key themes identified in the focus groups regarding facilitators of and barriers to accessing clinical prevention services*

\begin{tabular}{|c|c|c|}
\hline Level & Facilitator & Barrier \\
\hline \multirow[t]{6}{*}{ Patient } & $\begin{array}{l}\text { Taking responsibility for health } \\
(8,47)\end{array}$ & - \\
\hline & Knowledge $(8,83)$ & - \\
\hline & $\begin{array}{l}\text { Respect for advice of health } \\
\text { care professional }(6,31)\end{array}$ & - \\
\hline & - & Fear $(8,116)$ \\
\hline & - & Perceived low utility/risk $(6,38)$ \\
\hline & - & Side effects $(5,31)$ \\
\hline \multirow[t]{3}{*}{ Primary care provider } & $\begin{array}{l}\text { Active listening/unhurried } \\
\text { pace }(1,2)\end{array}$ & Rushing/not listening $(7,73)$ \\
\hline & Trusting relationship $(1,1)$ & Broken trust $(5,41)$ \\
\hline & - & Not sharing information $(6,48)$ \\
\hline \multirow[t]{5}{*}{ Health care system } & Embedded processes $(4,23)$ & No reminders $(4,11)$ \\
\hline & Availability of services $(5,30)$ & - \\
\hline & Low/no cost $(3,9)$ & - \\
\hline & - & $\begin{array}{l}\text { Limited time/1 issue per visit ( } 7 \text {, } \\
59)\end{array}$ \\
\hline & - & $\begin{array}{l}\text { Conflicting or changing } \\
\text { guidelines }(6,12)\end{array}$ \\
\hline
\end{tabular}


vider. In British Columbia, primary care physicians are remunerated predominantly under a fee-for-service model, which has been associated with short appointment times and office policies limiting the number of issues addressed per visit. ${ }^{27}$ Although this can be characterized as a well-known universal barrier ${ }^{28-32}$ and applies to the entire population, there is potential for a greater

Table 3: Illustrative quotes

\begin{tabular}{l}
\hline Facilitator \\
\hline Taking responsibility for health \\
"But if you know that this is the test you need, then you can go \\
and pressure the doctor. 'This is the test I need. This is my
\end{tabular}
right'." (Woman, group 2)

\section{Fear}

"So many of my friends won't get tests done because they think they'll be taken to the hospital. Some people live so many years without knowing about their illness and they are fine. As soon as they find out about it they begin to panic." (Woman, group 2)

"Some people think that if we go for the test ... we may get cancer." (Woman, group 7)

"He comes from Asia. ... We eat a lot of clarified butter, sweets; that's al fat. If he has knowledge - these are the benefits, these are the risks of taking, then he thinks he'll have to stop it, and then I don't know, he's scared that doctor would say - like, if it's there - then my sweets and everything will be stopped." (Man, group 8)

\section{Knowledge}

"Unless he doesn't know about his benefits or non-benefits, he won't accept it. Our people who come from India are new immigrants, they never used to bother. They would think 'I'm fine, I don't need this'." (Man, group 8)

"And examples. When somebody does it he was fine, and somebody didn't do it, what are the losses he had. If you give an example then it's more beneficial." (Man, group 8)

\section{Low utility/risk}

"Like, maybe I don't need one [the pneumococcal vaccine]. In India nobody is bothered about pneumonia. Older people, they don't give much importance to this." (Man, group 8 )

"There are so many injections like flu and for other things and so if you are healthy, then you don't care. 'I don't need those injections.' And sometimes there is information like those companies who make the medicine, they are ruining the economy." (Man, group 5)

\section{Trusting relationship}

"If the doctor establishes a good relationship with the patient and encourages him, then it's better for the patient." (Man, group 4)

\section{Unhurried pace}

"But now my doctor, he is really good. I've had the same doctor for the last seven, eight years and he's really good. He's a good doctor. I'm happy with the doctor now. So if I have any other problems, they should listen to each and every problem. Whatever problem you have, the doctor should listen to you." (Woman, group 2)

\section{Broken trust}

"If you will tell the doctor to check, only then they will check, otherwise they don't. The family doctor should check in routine the blood pressure, blood sugar. But they don't actually check the patient. They just prescribe the medicine, and they just ask verbally and write down the medicine and send the patient home." (Woman, group 7)

\section{Rushing/not listening}

"The doctor always will not talk to me, and then she will just write down the medicine for me. They don't have time to discuss with the patient. I show the list of my questions to the doctor and the doctor just gives me the medicine ... she didn't have time to discuss or ask about that."

(Woman, group 6)

"In India when we go to the doctor, they attend them properly. They listen to the patient in detail, but here they don't. They just write down the medicine. Send the patients home. Whenever I went to the clinic, doctor has never checked my diabetes sugar level. I just test myself. They don't. The family doctor does not do that. He should do, it's his duty, but he does not listen. So what is the use of the family doctor?" (Woman, group 7)

\section{Integrated processes}

"Every driver will go do the medical test ... he will have to go to this one [to maintain his class 4 driver's license]." (Man, group 4)

\section{Lack of reminders}

"You are advising us that after 50, we should go for the colon screening test. Why the doctors are not referring our cases to the specialist for all these tests? It's the doctor's duty. If you say that after 50 years of age every lady should go for colon test, so why the doctors are not ... referring their cases?" (Woman, group 7)

\section{Availability of services}

"At the Indo-Canadian senior centre, the nurse used to come and then they came to know about blood pressure. They should be in that kind of facility and when seniors can go and check their blood pressure, they can get all the information." (Woman, group 6)

\section{Short visit duration}

"But the doctor should also take care of the patients. There should be more clinics, and they should listen to the patients. The doctor should give sufficient time to each and every patient. The family doctor does not have this much of time to attend sufficient time." (Woman, group 7) "If I'm talking about stomach illnesses, flu, then he said, 'No, you come tomorrow. Come tomorrow, the day after too.' They don't want to listen to the other things, and they'll write down afterwards they just talked about one thing. One to 2 more topics, nothing more than.... [Over the course of multiple visits] my interest to get some knowledge will go away." (Man group 8) 


\section{OPEN}

negative effect on populations who may already have difficulty accessing care (e.g., immigrants, older people), and our participants clearly identified this barrier as a priority for intervention. Moreover, the role of physicians in the South Asian population is culturally significant, and the population places high value on the "physician directive" (confirmed by A.G.). This means that when there is limited time during visits for prevention activities, it may have a greater impact for South Asian people, as they expect leadership from their physician.

\section{Limitations}

Our study had low representation from adults aged 40-65. Our recruitment strategy focused on health care and community settings such as older-adult housing, day programs and health education events, all of which favour an older population. In addition, the timing of the focus groups coincided with patterns of seasonal travel to India, which led to attrition of potential participants and may have biased the sample. As such, this study does not fully reflect all South Asian populations in Surrey. Moreover, the small sample may have precluded gaining the full range of community perspectives, and the change in study design prevented intended comparisons between age groups.

In addition, Surrey has a relatively high concentration of South Asian health care providers. Questions in the demographic interview about whether participants were attached to a primary care provider and the language of that provider would have helped to better understand the study sample and perhaps explain why factors like provider language and culture were not often raised in this study. Surrey physicians are also remunerated predominantly via a fee-for-service model, which may limit the applicability of our results to other health care system contexts.

This study also excluded the provider perspective. This perspective was not the focus of the study, but our findings highlight the importance of the patient-provider interaction, and gaining the provider perspective will be essential before action is taken on the results of the study.

Finally, both coders were female and neither had a medical background, which may have influenced their coding approach and the study results. However, having 1 coder with a South Asian background was an asset, and presenting early results to the broader team for discussion ensured the interpretation of the results was culturally appropriate and resonated with both health authority and community service provider perspectives.

\section{Conclusion}

Our results highlight barriers to uptake of clinical prevention services in the health care system, provider and patient/community contexts. Based on these barriers, recommendations for practice include:

- In the context of the health care system, policies and remuneration methods that facilitate the inclusion of prevention in primary care (e.g., population-based funding and interdisciplinary primary care models) should be considered as a mechanism to increase the duration, comprehensiveness and prevention orientation of primary care visits. Policies and programs that enable integration of prevention into the health care and other systems along with reminders are also recommended.

- In the provider context, initiatives that promote ongoing relationships between patients and providers should be explored. However, our findings suggest that having an ongoing provider may not be sufficient to support uptake of prevention services without explicit attention to promoting communication and trust between patients and providers.

- In the patient/community context, outreach education and services are recommended and should be developed and tested in collaboration with the South Asian community.

Future research should test the impact of the suggested changes on uptake of clinical prevention services and population health outcomes. Moreover, the research should be embedded in the health care system and should use a participatory design to facilitate integrated knowledge translation, feasibility and sustainability.

\section{References}

1. Kendall PRW. Investing in prevention: improving health and creating sustainability. Victoria: Office of the Provincial Health Officer; 2010. Available: www. health.gov.bc.ca/library/publications/year/2010/Investing_in_prevention_ improving_health_and_creating_sustainability.pdf (accessed 2012 May 11).

2. Partners in prevention: implementing a lifetime prevention plan. Vancouver: British Columbia Medical Association; 2010. Available: www.doctorsofbc.ca/sites/ default/files/prevention_jun2010.pdf (accessed 2012 Feb. 16).

3. A lifetime of prevention: the report of the Clinical Prevention Policy Review Committee. Clinical Prevention Policy Review Committee; 2009. Available: www. health.gov.bc.ca/library/publications/year/2009/CPPR_Lifetime_of_Prevention Report.pdf (accessed 2012 Feb. 16).

4. Hanson K, Montgomery P, Bakker D, et al. Factors influencing mammography participation in Canada: an integrative review of the literature. Curr Oncol 2009;16: 65-75.

5. Lu M, Moritz S, Lorenzette D, et al. A systematic review of interventions to increase breast and cervical cancer screening uptake among Asian women. BMC Public Health 2012;12:413.

6. Menon U, Szalacha L, Prabhughate A. Breast and cervical cancer screening among South Asian immigrants in the United States. Cancer Nurs 2012;35;278-87.

7. Gupta M, Singh N, Verma S. South Asians and cardiovascular risk: what clinicians should know. Circulation 2006;113:e924-9.

8. Leung G, Stanner S. Diets of minority ethnic groups in the UK: influence on chronic disease risk and implications for prevention. Nutr Bull 2011;36:161-98.

9. Surood S, Lai D. Impact of culture on use of Western health services by older South Asian Canadians. Can 7 Public Health 2010;101:176-80.

10. Jones CA, Nanji A, Mawani S, et al. Feasibility of community-based screening for cardiovascular disease risk in an ethnic community. The South Asian Cardiovascular Health Assessment and Management Program (SA-CHAMP). BMC Public Health 2013;13:160.

11. Ahmad F, Cameron JI, Stewart DE. A tailored intervention to promote breast cancer screening among South Asian immigrant women. Soc Sci Med 2005;60:575-86.

12. Bharmal N, Chaudhry S. Preventive health services delivery to South Asians in the United States. F Immigr Minor Health 2012;14:797-802.

13. Grewal S, Bottorf JL, Balneaves LG. Pap test screening clinic in a South Asian community of Vancouver, British Columbia: challenges to maintaining utilization. Public Health Nurs 2004;21:412-8.

14. Gupta A, Kumar A, Stewart DE. Cervical cancer screening among South Asian women in Canada: the role of education and acculturation. Health Care Women Int 2002;23:123-34

15. Lofters AK, Moineddin R, Hwang SW, et al. Predictors of low cervical cancer screening among immigrant women in Ontario, Canada. BMC Womens Health 2011;11:20.

16. Redwood-Campbell L, Fowler N, Laryea S, et al. 'Before you teach me, I cannot know': immigrant women's barriers and enablers with regard to cervical cancer screening among different ethnolinguistic groups in Canada. Can 7 Public Health 2011;102:230-4.

17. Sandelowski M. Focus on research methods: Whatever happened to qualitative description? Res Nurs Health 2000;23:334-40

18. Canadian Community Health Survey: annual component - 2014 questionnaire. Ottawa: Statistics Canada; 2014. Available: www23.statcan.gc.ca/imdb-bmdi/ instrument/3226_Q1_V11-eng.pdf (accessed 2014 Jan. 25).

19. Gourlay A, Mshana G, Birdthistle I, et al. Using vignettes in qualitative research to 
explore barriers and facilitating factors to the uptake of prevention of mother-tochild transmission services in rural Tanzania: a critical analysis. BMC Med Res Methodol 2014;14:21

20. National Household Survey (NHS) profile, 2011. Surrey (city), British Columbia [table]. Ottawa: Statistics Canada; 2013. Cat no 99-004-XWE. Available: www12.statcan.gc.ca/nhs-enm/2011/dp-pd/prof/index.cfm?Lang=E (accessed 2015 Sept. 4)

21. Jafri NF. The role of culture and health literacy in cancer screening practices among young, middle to upper middle-class Pakistani-American women [dissertation/thesis abstract]. New York: Teachers College, Columbia University; 2011.

22. Patel N, Stone MA, McDonough C, et al. Concerns and perceptions about necessity in relation to insulin therapy in an ethnically diverse UK population with type 2 diabetes: a qualitative study focusing mainly on people of South Asian origin. Diabetic Med 2015;32:635-44

23. Vida Estacio E, McKinley RK, Saidy-Khan S, et al. Health literacy: why it matters to South Asian men with diabetes. Prim Health Care Res Dev 2015;16:214-8.

24. Zou P, Parry M. Strategies for health education in North American immigrant populations. Int Nurs Rev 2012;59:482-8.

25. Kobayashi LC, Wardle J, von Wagner C. Limited health literacy is a barrier to colorectal cancer screening in England: evidence from the English Longitudinal Study of Ageing. Prev Med 2014;61:100-5.

26. Ahmed S, Shommu NS, Rumana N, et al. Barriers to access of primary healthcare by immigrant populations in Canada: a literature review. 7 Immigr Minor Health 2015 Sept. 12 [Epub ahead of print].

27. Léger PT. Physician payment mechanisms: an overview of policy options for Canada. Ottawa: Canadian Health Services Research Foundation; 2011. Available: www.cfhi-fcass.ca/sf-docs/default-source/hospital-funding-docs/CHSRF -LegerPhysicianRenumerationENG.pdf?sfvrsn=0 (accessed 2015 Aug. 14).

28. Ayres CG, Griffith HM. Perceived barriers to and facilitators of the implementation of priority clinical preventive services guidelines. Am 7 Manag Care 2007;13: $150-5$

29. Hudon E, Beaulieu M, Roberge D. Integration of the recommendations of the Canadian Task Force on Preventive Health Care: obstacles perceived by a group of family physicians. Fam Pract 2004;21:11-7.

30. Mirand AL, Beehler GP, Kuo CL, et al. Explaining the de-prioritization of primary prevention: physicians' perceptions of their role in the delivery of primary care. BMC Public Health 2003;3:15.

31. Rubio-Valera M, Pons-Vigués M, Martínez-Andrés M, et al. Barriers and facilitators for the implementation of primary prevention and health promotion activities in primary care: a synthesis through meta-ethnography. PLoS One 2014;9:e89554-e89554

32. Yarnall KSH, Pollak KI, Østbye T, et al. Primary care: Is there enough time for prevention? Am 7 Public Health 2003;93:635-41.
Affiliations: MPH practicum student, Fraser Health Authority, Surrey, BC, and MPH candidate, University of Waterloo (Majid), Waterloo, Ont.; Population Health Observatory (Douglas), Fraser Health Authority, Surrey, BC; Fraser Health Authority (Lee), Surrey, BC; Digital Emergency Medicine (Stacy, Ho) and Department of Emergency Medicine (Ho), Faculty of Medicine, University of British Columbia, and the University of British Columbia P.A. Woodward Instructional Resources Centre (Stacy, Ho), Vancouver, BC; South Asian Health Institute, Fraser Health Authority and Department of Pathology and Laboratory Medicine, Faculty of Medicine, University of British Columbia (Garg), New Westminster, BC

Contributors: Sanaa Majid worked on this research project during her practicum with the Fraser Health Authority. She brought her perspective as a member of the South Asian community to support culturally appropriate interpretation of the data during coding and drafted the manuscript. Rachel Douglas led the qualitative content analysis, contributed to interpretation of the results and revised the manuscript for important intellectual content. Victoria Lee contributed to the study design, and Victoria Lee, Elizabeth Stacy and Kendall Ho revised the manuscript for important intellectual content. Arun Garg provided a cultural context for both the study design and interpretation of the results, and revised the manuscript for important intellectual content. All of the authors approved the final version submitted for publication and agreed to act as guarantors of the work.

Funding: This study was supported by the Surrey Hospital and Outpatient Centre Foundation.

Acknowledgements: The authors acknowledge the generous support of Nicole S. Berry in the development of the study design and Laurie Goldsmith in the analysis of results and review of this article. Their expertise and advice were integral to the success of this project. The authors also acknowledge Navdeep Sandhu, Harsimranjit Sidhu, Navpreet Dhaliwal, Manpreet Dhaliwal, Anisha Takher, Ramneek Dhanoa, Adesh Vohora, Anita Bal, Rupinder Sidhu and Paige Sandher, who generously volunteered their time to support study implementation; without them this project would not have been possible.

Supplemental information: For reviewer comments and the original submission of this manuscript, please see www.cmajopen.ca/content/4/3/ E390/suppl/DC1 